\title{
Agar dilution and agar screen with cefoxitin and oxacillin: what is known and what is unknown in detection of meticillin-resistant Staphylococcus
} aureus

Correspondence

Leandro Reus Rodrigues Perez leandro.reus@gmail.com

Received 7 October 2006

Accepted 4 April 2008

\author{
Leandro Reus Rodrigues Perez, ${ }^{1,2}$ Cícero Dias ${ }^{2,3}$ \\ and Pedro Alves d'Azevedo ${ }^{1,3}$
}
${ }^{1}$ Post-Graduate Program in Medical Sciences, Fundação Faculdade Federal de Ciências Médicas de Porto Alegre, RS, Brazil
${ }^{2}$ Laboratory of Microbiology, Hospital Mãe de Deus, Porto Alegre, RS, Brazil
${ }^{3}$ Microbiology Department, Fundação Faculdade Federal de Ciências Médicas de Porto Alegre, Porto Alegre, RS, Brazil

\section{INTRODUCTION}

Meticillin-resistant Staphylococcus aureus (MRSA) remains the leading causative agent of hospital-acquired infections. In Brazil, about $40-60 \%$ of hospital staphylococcal infections (respiratory tract, urinary tract, blood, surgical wound, invasive infections and others) are caused by MRSA (Teixeira et al., 1995).

Meticillin-resistance is attributable to the mecA gene, encoding penicillin-binding protein (PBP) $2 \mathrm{a}$, which presents low affinity for $\beta$-lactam antimicrobials (Fuda et al., 2004). Heterogeneous resistance to meticillin occurs among $S$. aureus isolates due to variations in the expression of the $m e c A$ gene, or alteration of constitutive PBPs (Chambers, 2001). The Clinical and Laboratory Standards Institute (CLSI; formerly NCCLS) criteria have recently been modified to better define meticillin resistance (CLSI, 2006b).

The detection of the meticillin resistance represents a real challenge for the routine clinical microbiology laboratories since molecular methods, the gold standard, are not available for most medical institutions in Brazil. Thus, phenotypic methods for characterization of the resistance to meticillin are frequently evaluated (Skov et al., 2003; Cauwelier et al., 2004; Fernandes et al., 2005; Pottumarthy

Abbreviations: CLSI, Clinical and Laboratory Standards Institute; MRSA, meticillin-resistant $S$. aureus; PBP, penicillin-binding protein. et al., 2005; Sharp et al., 2005; Swenson \& Tenover, 2005; Velasco et al., 2005).

This study was designed to evaluate the performance of the oxacillin agar screen and the MIC values of oxacillin and cefoxitin antimicrobials as determined by agar dilution test, to predict resistance among $S$. aureus harbouring the $\operatorname{mec} A$ gene.

\section{METHODS}

Bacterial isolates. From August to December 2002, 167 S. aureus isolates were obtained from blood cultures of patients hospitalized at three hospitals in Porto Alegre, RS, Brazil. Only one isolate from each patient was included in the study.

Oxacillin agar screen. This test was carried out according to the CLSI guidelines (CLSI, 2006b). A McFarland 0.5 suspension was spotted onto Mueller-Hinton agar (Becton Dickinson) containing $4 \%$ (w/v) $\mathrm{NaCl}$ and $6 \mu$ oxacillin $\mathrm{ml}^{-1}$, and incubated at $35^{\circ} \mathrm{C}$ for $24 \mathrm{~h}$.

Determination of MIC. Susceptibility to oxacillin and cefoxitin was evaluated by the agar dilution method according to CLSI guidelines (CLSI, 2006a). The oxacillin and cefoxitin concentrations used ranged from 0.5 to $256 \mu \mathrm{g} \mathrm{ml}^{-1}$. Mueller-Hinton agar plates without antimicrobial were used as controls of bacterial growth.

Detection of the mecA gene by PCR. PCR was used to confirm the presence of the mecA gene. Primers mecA 1 (5'-TGG CTA TCG TGT CAC AAT CG) and $m e c A_{2}\left(5^{\prime}\right.$-CTG GAA CTT GTT GAG CAG AG) 
were used to amplify a $310 \mathrm{bp}$ segment of the gene; these were electrophoresed in a $1.5 \%$ agarose gel and visualized under UV light by the addition of ethidium bromide $\left(0.5 \mu \mathrm{g} \mathrm{ml}^{-1}\right)$ (Vannuffel et al., 1998).

Control strains. Meticillin-susceptible S. aureus (MSSA) ATCC 29213 and MRSA ATCC 33591 strains were used as controls in susceptibility tests and PCRs.

Statistical parameters. The concordance between MIC values of oxacillin and cefoxitin was calculated by Pearson's correlation coefficient $(r)$. The following value and correlation criteria were adopted: 0, null; 0-0.3, weak; 0.3-0.6, regular; 0.6-0.9, strong; 0.9-1, very strong; 1 , full or absolute.

\section{RESULTS AND DISCUSSION}

Presence of the mecA gene was observed in $41.1 \%(69 / 167)$ of the $S$. aureus isolates in this study. Since 2004, the CLSI guidelines have recognized the use of cefoxitin, a cephamycin, as a surrogate marker of meticillin resistance. However, the agar dilution method is only standardized for oxacillin; S. aureus isolates are characterized as resistant and susceptible with $\mathrm{MIC} \geqslant 4 \mu \mathrm{g} \mathrm{ml}^{-1}$ and $\leqslant 2 \mu \mathrm{g} \mathrm{ml} \mathrm{m}^{-1}$, respectively (CLSI, 2006b). Using these breakpoints, one isolate was mischaracterized as resistant ( $\mathrm{mec} A$-negative with an MIC of $4 \mu \mathrm{g}$ oxacillin $\mathrm{ml}^{-1}$ ) (Table 1). For cefoxitin, the best results were obtained with breakpoints of $\leqslant 4 \mu \mathrm{g} \mathrm{ml}^{-1}$ and $>4 \mu \mathrm{g} \mathrm{ml}^{-1}$ for susceptible and resistant, respectively (only one false-resistant isolate), with $99.4 \%$ accuracy. Skov et al. (2006) used a highly diverse collection of $S$. aureus isolates to perform a comprehensive investigation on test conditions to accurately define MRSA, and suggested interpretative criteria of $\leqslant 4 \mu \mathrm{g}$ cefoxitin $\mathrm{ml}^{-1}$ and $>4 \mu \mathrm{g}$ cefoxitin $\mathrm{ml}^{-1}$ for susceptible and resistant, respectively. However, Skov et al. (2006) used the Etest to determine MIC, and the breakpoint of $\leqslant 4 \mu \mathrm{g} \mathrm{ml}^{-1}$ and $>4 \mu \mathrm{g} \mathrm{ml}^{-1}$ resulted in one mecA-positive isolate being misclassified as susceptible. The breakpoint established in the CLSI guidelines for susceptibility testing using cefoxitin ( $\leqslant 8 \mu \mathrm{g} \mathrm{ml}^{-1}, 16 \mu \mathrm{g} \mathrm{ml}^{-1}$ and $\geqslant 32 \mu \mathrm{g} \mathrm{ml}^{-1}$ for susceptible, intermediate and resistant, respectively) presented low sensitivity for mecA gene detection and must not be utilized for this purpose (Table 1); this was also evident in the study by Skov et al. (2006).

The oxacillin agar screen test showed 98.5\% (68/69), $100 \%$ $(98 / 98)$ and $99.4 \%(166 / 167)$ sensitivity, specificity and accuracy, respectively; it is therefore highly reliable in discriminating isolates harbouring the mecA gene (only one false-susceptible result was obtained).

A potential limitation of this study lies in the fact that the genetic background of the isolates is not clearly defined. In Brazil, a specific clone predominates among MRSA (Amaral et al., 2005), and at least one additional distinct clone was observed when nine randomly selected isolates were submitted to PFGE analysis after restriction with SmaI (data not shown). The significance of our findings may therefore be limited by possible over-representation of a few MRSA clones.
Table 1. Detection of meticillin resistance among $S$. aureus isolates by presence of the $\operatorname{mec} A$ gene, and MIC of cefoxitin and oxacillin

\begin{tabular}{|c|c|c|c|c|}
\hline \multirow{2}{*}{$\begin{array}{l}\text { Breakpoint } \\
\left(\mu \mathrm{g} \mathrm{ml}^{-1}\right)^{\star}\end{array}$} & \multicolumn{2}{|c|}{ Cefoxitin } & \multicolumn{2}{|c|}{ Oxacillin } \\
\hline & $\begin{array}{c}\text { mecA- } \\
\text { negative } \dagger \\
(n=98)\end{array}$ & $\begin{array}{c}\text { mecA- } \\
\text { positive } \dagger \\
(n=69)\end{array}$ & $\begin{array}{c}\text { mecA- } \\
\text { negative } \dagger \\
(n=98)\end{array}$ & $\begin{array}{c}\text { mec } A- \\
\text { positive } \dagger \\
(n=69)\end{array}$ \\
\hline$\leqslant 0.25$ & 0 & 0 & 15 & 0 \\
\hline 0.5 & 1 & 0 & 12 & 0 \\
\hline 1 & 23 & 0 & 36 & 0 \\
\hline 2 & 68 & 0 & 34 & 0 \\
\hline 4 & 5 & 0 & 1 & 0 \\
\hline 8 & 1 & 6 & 0 & 7 \\
\hline 16 & 0 & 7 & 0 & 2 \\
\hline 32 & 0 & 3 & 0 & 1 \\
\hline 64 & 0 & 3 & 0 & 22 \\
\hline 128 & 0 & 34 & 0 & 19 \\
\hline$\geqslant 256$ & 0 & 16 & 0 & 18 \\
\hline
\end{tabular}

${ }^{*}$ Cefoxitin and oxacillin MICs were determined by an agar dilution method (CLSI, 2006a).

$\dagger$ Presence of mecA was determined by PCR.

The Pearson's correlation coefficient (Fig. 1) shows that there is a strong association between cefoxitin and oxacillin MIC values $(r=0.73)$. This means that the expression of resistance or susceptibility is comparable for the two antimicrobial agents when the same technique and conditions are adopted. Either drug may be used as a surrogate for testing meticillin resistance when an adequate methodology is used.

Finally, our results showed that the best method for predicting resistance mediated by the mecA gene was obtained by the agar dilution test using oxacillin (breakpoint of $4 \mu \mathrm{g} \mathrm{ml}^{-1}$ ), cefoxitin (breakpoint of $8 \mu \mathrm{g} \mathrm{ml}^{-1}$ ) or the oxacillin agar screen ( $99.4 \%$ accuracy).

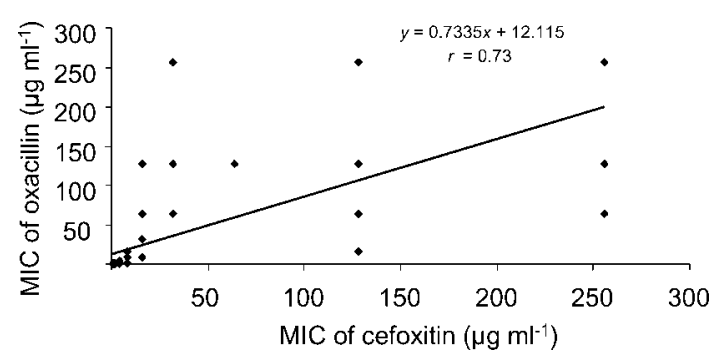

Fig. 1. Correlation between cefoxitin and oxacillin MIC values of $S$. aureus. MIC values were obtained by agar dilution testing of $S$. aureus strains as described by CLSI (2006a). Pearson's correlation coefficient was used to determine concordance between these two methods. 


\section{REFERENCES}

Amaral, M. M., Coelho, L. R., Flores, R. P., Souza, R. R., SilvaCarvalho, M. C., Teixeira, L. A., Ferreira-Carvalho, B. T. \& Figueiredo, A. M. (2005). The predominant variant of the Brazilian epidemic clonal complex of methicillin-resistant Staphylococcus aureus has an enhanced ability to produce biofilm and to adhere to and invade airway epithelial cells. J Infect Dis 192, 801-810.

Cauwelier, B., Gordts, B., Descheemaecker, P. \& Van Landuyt, H. (2004). Evaluation of a disk diffusion method with cefoxitin (30 microg) for detection of methicillin-resistant Staphylococcus aureus. Eur J Clin Microbiol Infect Dis 23, 389-392.

Chambers, H. F. (2001). The changing epidemiology of Staphylococcus aureus? Emerg Infect Dis 7, 178-182.

CLSI (2006a). Methods for Dilution Antimicrobial Susceptibility Tests for Bacteria that Grow Aerobically. Approved standard, CLSI document M7-A7. Wayne, PA: Clinical and Laboratory Standards Institute.

CLSI (2006b). Performance Standards for Antimicrobial Susceptibility Testing. Sixteenth Informational Supplement. Wayne, PA: Clinical and Laboratory Standards Institute.

Fernandes, C. J., Fernandes, L. A. \& Collignon, P. (2005). Cefoxitin resistance as a surrogate marker for the detection of methicillinresistant Staphylococcus aureus. J Antimicrob Chemother 55, 506-510.

Fuda, C., Suvorov, M., Vakulenko, S. B. \& Mobashery, S. (2004). The basis for resistance to beta-lactam antibiotics by penicillin-binding protein 2a of methicillin-resistant Staphylococcus aureus. J Biol Chem 279, 40802-40806.

Pottumarthy, S., Fritsche, T. R. \& Jones, R. N. (2005). Evaluation of alternative disk diffusion methods for detecting mecA-mediated oxacillin resistance in an international collection of staphylococci: validation report from the SENTRY Antimicrobial Surveillance Program. Diagn Microbiol Infect Dis 51, 57-62.

Sharp, S. E., Warren, J. A. \& Thomson, R. B., Jr (2005). Cefoxitin disk diffusion screen for confirmation of oxacillin-resistant Staphylococcus aureus isolates and utility in the clinical laboratory. Diagn Microbiol Infect Dis 51, 69-71.

Skov, R., Smyth, R., Clausen, M., Larsen, A. R., Frimodt-Moller, N., Olsson-Liljequist, B. \& Kahlmeter, G. (2003). Evaluation of a cefoxitin 30 microg disc on Iso-Sensitest agar for detection of methicillin-resistant Staphylococcus aureus. J Antimicrob Chemother 52, 204-207.

Skov, R., Smyth, R., Larsen, A. R., Bolmstrôm, A., Karlsson, A., Mills, K., Frimodt-Moller, N. \& Kahlmeter, G. (2006). Phenotypic detection of methicillin resistance in Staphylococcus aureus by disk diffusion testing and Etest on Mueller-Hinton agar. J Clin Microbiol 44, 4395-4399.

Swenson, J. M. \& Tenover, F. C. (2005). Results of disk diffusion testing with cefoxitin correlate with presence of mecA in Staphylococcus spp. J Clin Microbiol 43, 3818-3823.

Teixeira, L. A., Resende, C. A., Ormonde, L. R., Rosenbaum, R., Figueiredo, A. M., de Lencastre, H. \& Tomasz, A. (1995). Geographic spread of epidemic multiresistant Staphylococcus aureus clone in Brazil. J Clin Microbiol 33, 2400-2404.

Vannuffel, P., Laterre, P. F., Bouyer, M., Gigi, J., Vandercam, B., Reynaert, M. \& Gala, J. L. (1998). Rapid and specific molecular identification of methicillin-resistant Staphylococcus aureus in endotracheal aspirates from mechanically ventilated patients. J Clin Microbiol 36, 2366-2368.

Velasco, D., del Mar Tomas, M., Cartelle, M., Beceiro, A., Perez, A., Molina, F., Moure, R., Villanueva, R. \& Bou, G. (2005). Evaluation of different methods for detecting methicillin (oxacillin) resistance in Staphylococcus aureus. J Antimicrob Chemother 55, 379-382. 